\title{
Relativistic Corrections to the Aharonov-Bohm Scattering
}

\author{
M. Gomes, J. M. C. Malbouisson* and A. J. da Silva \\ Instituto de Física, Universidade de São Paulo, Caixa Postal 66318, \\ 05315-970, São Paulo, SP, Brazil.
}

\begin{abstract}
We determine the $|\mathbf{p}| / m$ expansion of the two body scattering amplitude of the quantum theory of a Chern-Simons field minimally coupled to a scalar field with quartic self-interaction. It is shown that the existence of a critical value of the self-interaction parameter for which the 2-particle amplitude reduces to the Aharonov-Bohm one is restricted to the leading, nonrelativistic, order. The subdominant terms correspond to relativistic corrections to the Aharonov-Bohm scattering.
\end{abstract}

PACS: 03.65.Bz,03.70.+k,11.10.Kk

\section{INTRODUCTION}

The Aharonov-Bohm (AB) effect [1], the scattering of charged particles by a flux line, is one of the most fascinating problems of planar nonrelativistic (NR) quantum dynamics which has recently regained great interest due to its connection to the physics of anyons [2⿴囗十. It has exact solution but if the scattering potential is treated as a perturbation, due to its singular nature, divergences occur in the perturbative series and so an appropriate renormalization is necessary to get the correct expansion. Moreover, through perturbative

\footnotetext{
*On leave of absence from Instituto de Física, Universidade Federal da Bahia, Salvador, 40210-340, Brazil.
} 
renormalization, the $\mathrm{AB}$ potential becomes related to the delta function, contact, interaction. These issues have been discussed from the quantum mechanical (first-quantized) viewpoint [5] and also by using a field theoretical (second-quantized) approach.

In the latter case, one considers a NR scalar field minimally coupled to a Chern-Simons (CS) gauge field [6] and includes a quartic self-interaction [7] in such a manner that the Galilean invariant Lagrangian density [8] is given by

$$
\mathcal{L}_{N R}=\psi^{*}\left(i D_{t}+\frac{\mathbf{D}^{2}}{2 m}\right) \psi-\frac{v_{0}}{4}\left(\psi^{*} \psi\right)^{2}+\frac{\Theta}{2} \partial_{t} \mathbf{A} \times \mathbf{A}-\Theta A_{0} \nabla \times \mathbf{A}
$$

This Lagrangian deeply differs from a relativistic one in the particle kinetics which is manifest in the NR free particle propagator $\Delta_{N R}(\omega, \mathbf{k})=i\left[\omega-\mathbf{k}^{2} / 2 m+i \varepsilon\right]^{-1}$.Up to one loop, the 2-particle scattering amplitude, calculated in the center of mass (CM) frame, is

$$
\mathcal{A}_{N R}^{(1)}=-v_{0}-i \frac{2 e^{2}}{m \Theta} \cot \theta+\frac{m}{8 \pi}\left(v_{0}^{2}-\frac{4 e^{4}}{m^{2} \Theta^{2}}\right)\left[\ln \left(\frac{\Lambda_{N R}^{2}}{\mathbf{p}^{2}}\right)+i \pi\right],
$$

where $\Lambda_{N R}$ is a nonrelativistic ultraviolet cutoff . The renormalization is implemented by redefining the self-coupling constant, $v_{0}=v+\delta v$, so that the total renormalized nonrelativistic amplitude is given, up to order $e^{4}$, by

$$
\mathcal{A}_{N R}=-v-i \frac{2 e^{2}}{m \Theta} \cot \theta+\frac{m}{8 \pi}\left(v^{2}-\frac{4 e^{4}}{m^{2} \Theta^{2}}\right)\left[\ln \left(\frac{\mu^{2}}{\mathbf{p}^{2}}\right)+i \pi\right],
$$

where $\mu$ is an arbitrary mass scale, introduced by the renormalization, that breaks the scale invariance of the amplitude $[8]$.

At the critical values $v_{c}^{ \pm}= \pm 2 e^{2} / m|\Theta|$, the one loop scattering vanishes, scale invariance is restored and by choosing the $v_{c}^{+}$value, corresponding to a repulsive contact interaction, the amplitude reduces, after multiplying by the appropriate kinematical factor, to the AharonovBohm amplitude for identical particles which is given by [1, 8]

$$
\mathcal{F}_{\mathrm{AB}}(|\mathbf{p}|, \theta)=-i \sqrt{\frac{\pi}{|\mathbf{p}|}} \alpha[\cot \theta-i \operatorname{sgn}(\alpha)]+\mathcal{O}\left(\alpha^{3}\right)
$$

where $\alpha=e^{2} / 2 \pi \Theta$. The existence of scale invariance at the critical self-coupling has been explicitly verified up to three loop level using differential regularization [9] and was recently 
proven to hold in all orders of perturbation theory [10]. Also, starting from the relativistic action for scalar self-interacting field minimally coupled to a CS gauge field, it was shown that the nonrelativistic limit of the one loop renormalized 2-particle scattering reduces to the nonrelativistic scale invariant one for the same critical values [11].

This letter is concerned with the question to what extent the relativistic corrections preserve such criticality. Using an intermediate cutoff procedure [12], which allows the determination of the $|\mathbf{p}| / m$ expansion of the quantum amplitudes, we calculate the 1-loop particle-particle CM scattering amplitude, for low external momenta, up to order $\mathbf{p}^{2} / \mathrm{m}^{2}$. The leading term of the $|\mathbf{p}| / m$ expansion coincides with the result of Ref. [11] whereas the subdominant parts do not vanish at the critical self-interaction values and so represent relativistic corrections to the Aharonov-Bohm scattering.

\section{THE RELATIVISTIC MODEL}

We consider a charged self-interacting scalar field in $2+1$ dimensions minimally coupled to a Chern-Simons gauge field described by the Lagrangian density

$$
\mathcal{L}=\left(D_{\mu} \phi\right)^{*}\left(D^{\mu} \phi\right)-m^{2} \phi^{*} \phi-\frac{\lambda}{4}\left(\phi^{*} \phi\right)^{2}+\frac{\Theta}{2} \epsilon_{\sigma \mu \nu} A^{\sigma} \partial^{\mu} A^{\nu}-\frac{\xi}{2}\left(\partial_{i} A^{i}\right)^{2}
$$

where $D_{\mu}=\partial_{\mu}-i e A_{\mu}$ is the covariant derivative, $\epsilon_{\sigma \mu \nu}$ is the fully antisymmetric tensor normalized to $\epsilon_{012}=+1$, the Minkowski metric signature is $(1,-1,-1)$, the units are such that $\hbar=c=1$ and repeated greek indices sum from 0 to 2 while repeated latin indices sum from 1 to 2. The choice of the Coulomb gauge fixing, the same used in Ref. [8], is dictated by convenience in discussing the nonrelativistic limit. It generates, in the Landau limit $(\xi \rightarrow \infty)$, a virtual gauge field propagator which is independent of $k^{0}$ and totally antisymmetric in the Minkowski indices with the only nonvanishing components given by

$$
D_{0 i}(k)=-D_{i 0}(k)=\frac{1}{\Theta} \frac{\epsilon_{i j} k^{j}}{\mathbf{k}^{2}}
$$

where $\epsilon_{i j}=\epsilon_{0 i j}$. The free propagator of the bosonic matter field is the usual Feynman propagator $\Delta(p)=i\left[p^{2}-m^{2}+i \varepsilon\right]^{-1}$ and the vertex factors are $-i \lambda$ for the self-interaction 
vertex and $-i e\left(p+p^{\prime}\right)^{\mu}$ and $2 i e^{2} g^{\mu \nu}$ for the trilinear and the seagull vertices that always arise from minimal coupling with a scalar field. With $V$ and $N$ denoting the numbers of vertices and external lines, the degree of superficial divergence of a generic graph is given by $d(G)=3-\frac{1}{2} N_{\phi}-N_{A}-V_{\phi^{4}}$ and, thus, we must expect to face divergences in calculating radiative corrections. In the sequel we shall analyse these corrections at one loop level.

Owing to charge conjugation and the antisymmetry of the gauge field propagator (2.2), the only non-vanishing contribution to the 1-loop particle self-energy is an infinite constant coming from the tadpole graph which can be absorbed into the definition of the physical mass $m$.

The vacuum polarization shown in Fig. 1 is, naturally, independent of the gauge fixing chosen and dimensional renormalization gives

$$
\Pi_{\mu \nu}^{(1)}(q)=-\frac{i e^{2}}{2 \pi} m\left(1-\int_{0}^{1} d x \sqrt{1-q^{2} x(1-x) / m^{2}}\right) \frac{1}{q^{2}}\left[q^{2} g_{\mu \nu}-q_{\mu} q_{\nu}\right]
$$

which reduces, in the low momentum regime $\left|q^{2}\right| \ll m^{2}$, to

$$
\Pi_{\mu \nu}^{(1)}(q) \simeq-\frac{i e^{2}}{24 \pi m}\left(1+\frac{q^{2}}{20 m^{2}}\right)\left[q^{2} g_{\mu \nu}-q_{\mu} q_{\nu}\right]
$$

It should be noticed that using a cutoff regulator one gets explicitly the linearly divergent part but the finite one is the same as above.

The 1-loop correction to the trilinear gauge coupling vertex, shown in Fig. 2, is finite. (The other possible 1-loop contribution, which has one trilinear and one self-interaction vertex inserted in a particle loop, is null by charge conjugation.) The sum of the two first parcels, the contribution involving the seagull vertex, is exactly given, for external particles legs in the mass shell, by

$$
\Gamma_{3(S)}^{(1) 0}=0 \text { and } \Gamma_{3(S)}^{(1) l}=\frac{-e^{3}}{2 \pi \Theta} \epsilon_{i j} g^{j l}\left[\frac{p^{i} \sqrt{m^{2}+\mathbf{p}^{2}}}{\sqrt{m^{2}}+\sqrt{m^{2}+\mathbf{p}^{2}}}-\frac{p^{i} \sqrt{m^{2}+\mathbf{p}^{\prime 2}}}{\sqrt{m^{2}}+\sqrt{m^{2}+\mathbf{p}^{\prime 2}}}\right] \text {. }
$$

On the other hand, the triangle graph can be expressed as

$$
\Gamma_{3(T)}^{(1) \mu}=\frac{e^{3}}{8 \pi \Theta} \int_{0}^{1} d x \int_{0}^{1} d y\left[\frac{A^{\mu}}{\left(Q_{0}^{2}-y \mathbf{Q}^{2}\right)^{3 / 2}}+\frac{B^{\mu}}{\left(Q_{0}^{2}-y \mathbf{Q}^{2}\right)^{1 / 2}}\right]
$$


with the numerators given by $A^{0}=\epsilon_{i j} p^{i} q^{j} Q^{0}\left(P^{0}-2 Q^{0}\right), A^{l}=\epsilon_{i j} p^{i} q^{j} Q^{0}\left(P^{l}-2 y Q^{l}\right), B^{0}=$ $\epsilon_{i j} p^{i} q^{j}(-2)$ and $B^{l}=\epsilon_{i j}\left(4 V^{i}+2 Q^{0} q^{i}\right) g^{j l}$ where $q^{\mu}=p^{\mu}-p^{\prime \mu}, P^{\mu}=p^{\mu}+p^{\prime \mu}, V^{i}=q^{0} p^{i}-p^{0} q^{i}$ and $Q^{\mu}(x)=p^{\mu}-q^{\mu}(1-x)$. The $y$ integration in (2.6) is easy but the remaining $x$ one is very complicated. For sake of simplicity, we restrict ourselves to the situation where $p^{0}=p^{0}$ which the relevant one for the calculation of the 1-loop CM two body scattering. In this case, $Q_{0}^{2}=m^{2}+\mathbf{p}^{2}$ and expanding (2.5) and (2.6) for $|\mathbf{p}| / m$ small one obtains the total trilinear vertex correction, up to order $\mathbf{p}^{2} / \mathrm{m}^{2}$, as

$$
\left.\Gamma_{3}^{(1) 0}(p, p-q)\right|_{q^{0}=0} \simeq \frac{-e^{3}}{4 \pi \Theta}\left[\frac{\epsilon_{i j} p^{i} q^{j}}{m}\right]
$$

and

$$
\left.\Gamma_{3}^{(1) l}(p, p-q)\right|_{q^{0}=0} \simeq \frac{-e^{3}}{4 \pi \Theta}\left[\epsilon_{i j} q^{i} g^{j l}\right]\left(2+\frac{1}{12}(5+\cos \theta) \frac{\mathbf{p}^{2}}{m^{2}}\right)+\frac{e^{3}}{4 \pi \Theta}\left[\frac{\epsilon_{i j} p^{i} q^{j}}{m}\right] \frac{\left(p+p^{\prime}\right)^{l}}{4 m},
$$

where $\theta$ is the angle between the vectors $\mathbf{p}$ and $\mathbf{p}^{\prime}$. In the above equations, and from now on, the symbol $\simeq$ denotes that the expression which follows holds up to the order $\mathbf{p}^{2} / \mathrm{m}^{2}$.

The one loop correction to the seagull vertex is of fourth order in the charge $e$, and so it does not enter in the particle-particle scattering at one loop level. The correction to the self-interaction vertex, which actually represents the 2-particle scattering in one loop order, will be calculated in the next section.

\section{PARTICLE-PARTICLE SCATTERING}

In the CM frame, with external particles on the mass shell, one has $\mathbf{p}_{1}=-\mathbf{p}_{2}=\mathbf{p}$, $\mathbf{p}_{1}^{\prime}=-\mathbf{p}_{2}^{\prime}=\mathbf{p}^{\prime}$ and $p_{1}^{0}=p_{2}^{0}=p_{1}^{\prime 0}=p_{2}^{\prime 0}=w_{p}=\sqrt{m^{2}+\mathbf{p}^{2}}$. The tree level particle-particle amplitude, presented in Fig. 3, is given by

$$
A^{(0)}=-\lambda-i \frac{8 e^{2}}{\Theta} \sqrt{m^{2}+\mathbf{p}^{2}} \cot \theta \simeq-\lambda-i \frac{8 e^{2}}{\Theta} m\left(1+\frac{\mathbf{p}^{2}}{2 m^{2}}\right) \cot \theta,
$$

where $\theta$ is the scattering angle and $m$ is the renormalized mass of the bosonic particle. One sees that, by definiteness, we take the amplitude as being $(-i)$ times the 1PI four 
point function. This choice is only to facilitate the comparison with the nonrelativistic case discussed in ref. [8].

We shall calculate the 1-loop order scattering amplitude, for low external momenta and up to order $\mathbf{p}^{2} / \mathrm{m}^{2}$. The insertion of the vacuum polarization into the tree level gives, using (2.4) with $q^{0}=0$, the contribution

$$
A^{(p)} \simeq-\frac{2 e^{4}}{\pi \Theta^{2}} m\left\{\frac{1}{6}+\frac{7}{30} \frac{\mathbf{p}^{2}}{m^{2}}\right\} .
$$

Similarly, the trilinear vertex correction $\Gamma_{3}^{(1) \mu}$, given by (2.7) and (2.8), inserted into the tree level leads to the following $e^{4}$-contribution

$$
A^{(v)} \simeq-\frac{2 e^{4}}{\pi \Theta^{2}} m\left\{2+\frac{5}{3} \frac{\mathbf{p}^{2}}{m^{2}}\right\} .
$$

Diagrams, like those shown in Fig. 4, that admixes particle self-interaction and gauge field exchange, do not contribute. The first vanishes by charge conjugation, the second is null due to the antisymmetric form of the gauge field propagator whereas the chalice diagram is proportional to $\sin \theta$ and, thus, is eliminated by its final particles exchanged partner. The most important, genuine, one loop particle-particle scattering comes from the diagrams shown in Fig. 5 where it is also presented the routing of external momenta used in the calculations.

The group $(a)$ is the finite self-interaction scattering, which can be exactly calculated $11-12$ and is given by

$$
\begin{aligned}
A^{(a)}= & \frac{\lambda^{2}}{32 \pi m}\left\{\frac{1}{\sqrt{1+\mathbf{p}^{2} / m^{2}}}\left[\ln \left(\frac{\sqrt{1+\mathbf{p}^{2} / m^{2}}+1}{\sqrt{1+\mathbf{p}^{2} / m^{2}}-1}\right)+i \pi\right]\right. \\
& \left.+\left[\frac{2}{\sqrt{(1-\cos \theta) \mathbf{p}^{2} / 2 m^{2}}} \arctan \left(\sqrt{(1-\cos \theta) \mathbf{p}^{2} / 2 m^{2}}\right)+(\theta \rightarrow \pi-\theta)\right]\right\} \\
\simeq & \frac{\lambda^{2}}{32 \pi m}\left\{\left(1-\frac{\mathbf{p}^{2}}{2 m^{2}}\right)\left[\ln \left(\frac{4 m^{2}}{\mathbf{p}^{2}}\right)+i \pi\right]+4-\frac{\mathbf{p}^{2}}{6 m^{2}}\right\} .
\end{aligned}
$$

The $|\mathbf{p}| / m$ expansion of the more involving CS scattering, the $(b)$ and $(c)$ groups of diagrams of Fig. 5, will be calculated employing the following cutoff procedure [12]. 
First of all, we integrate over $k^{0}$ (the frequency part of the loop momentum $k$ ) without making any restriction in order to guarantee locality in time. This integration is greatly facilitated in the gauge we are working since the gauge field propagator does not depend on $k^{0}$. The remaining integration over the Euclidean $\mathbf{k}$ plane is then separated into two parcels through the introduction of an intermediate cutoff $\Lambda_{I}$ in the $|\mathbf{k}|$ integration satisfying

$$
\text { (i) }|\mathbf{p}| \ll \Lambda_{I} \ll m \text { and } \quad(i i)\left(\frac{|\mathbf{p}|}{\Lambda_{I}}\right)^{2} \approx\left(\frac{\Lambda_{I}}{m}\right)^{2} \approx \frac{|\mathbf{p}|}{m} .
$$

Condition $(i)$ presupposes that nonrelativistic spatial momenta are indeed much smaller than the particle mass while condition $(i i)$ establishes $\eta \approx|\mathbf{p}| / m$ as the small expansion parameter. The auxiliary cutoff $\Lambda_{I}$ splits the space of the intermediate states into two parts, the low (L) energy sector $\left(|\mathbf{k}|<\Lambda_{I}\right)$ and the high $(\mathrm{H})$ energy one with $|\mathbf{k}|>\Lambda_{I}$. In the $\mathrm{L}$ sector all the spatial momenta involved are small $(|\mathbf{p}| / m,|\mathbf{k}| / m \ll 1)$ and so one can perform a $1 / m$ expansion of the integrand while, in the $H$ - region, $|\mathbf{k}| \gg|\mathbf{p}|$ and the integrand can be expanded in a Taylor series around $|\mathbf{p}|=0$ and then, in both cases, integrated term by term (a regularization scheme has to be used if the graph is ultraviolet divergent). This procedure permits analytical calculations in every order in $\eta$, produces $\Lambda_{I^{-}}$dependent results and further expansions in $\Lambda_{I} / m$ may be necessary to get the $|\mathbf{p}| / m$ expansion of the $\mathrm{L}$ and $\mathrm{H}$ contributions to the amplitude, up to the desired order. Certainly, for sake of consistence, the $\Lambda_{I^{-}}$dependent parcels of the $\mathrm{L}$ and the $\mathrm{H}$ contributions of each diagram cancel identically. This process has been explicitly verified to produce the correct $|\mathbf{p}| / m$ expansion for the self-interaction scattering $(a)$ [12].

Consider the "right" box diagram corresponding to the direct exchange of two virtual gauge particles, the first parcel of Fig. 5(b). Following the Feynman rules, one has

$$
\begin{aligned}
A^{(b) d i r}= & -i e^{4} \int \frac{d^{3} k}{(2 \pi)^{3}}\left\{\left(p_{1}+k\right)^{\mu} D_{\mu \sigma}\left(k-p_{1}\right)\left(2 p_{2}+p_{1}-k\right)^{\sigma} \Delta(k)\right. \\
& \left.\Delta\left(p_{1}+p_{2}-k\right)\left(-k+p_{1}+p_{2}+p_{2}^{\prime}\right)^{\rho} D_{\rho \nu}\left(k-p_{1}^{\prime}\right)\left(k+p_{1}^{\prime}\right)^{\nu}\right\}+\left[p_{1}^{\prime} \leftrightarrow p_{2}^{\prime}\right] \\
= & -\frac{4 e^{4}}{\pi^{2} \Theta^{2}} \int d^{2} \mathbf{k}\left(\frac{w_{p}^{2}}{w_{k}}\right) \frac{1}{\mathbf{p}^{2}-\mathbf{k}^{2}+i \varepsilon}\left[\frac{(\mathbf{k} \times \mathbf{p})\left(\mathbf{k} \times \mathbf{p}^{\prime}\right)}{(\mathbf{k}-\mathbf{p})^{2}\left(\mathbf{k}-\mathbf{p}^{\prime}\right)^{2}}\right]+\left[\mathbf{p}^{\prime} \leftrightarrow-\mathbf{p}^{\prime}\right],
\end{aligned}
$$

where the $k^{0}$ integration was done as a contour integral. The angular integration in the last 
line above can be cast in the form $\frac{1}{2}\left[\cos \theta \mathrm{I}_{0}-\mathrm{I}_{2}\right]$ where

$$
\mathrm{I}_{n}=\int_{0}^{2 \pi} d \varphi \frac{\cos (n \varphi)}{[2 \cos (\varphi-\theta / 2)-\beta][2 \cos (\varphi+\theta / 2)-\beta]}
$$

and $\beta=\left(\mathbf{k}^{2}+\mathbf{p}^{2}\right) /(|\mathbf{k}||\mathbf{p}|)$. This integral can be done using the residue theorem and one finds

$$
\begin{aligned}
A^{(b) d i r}=-\frac{e^{4}}{\pi \Theta^{2}} \int d\left(\mathbf{k}^{2}\right) & \left(\frac{w_{p}^{2}}{w_{k}}\right) \frac{1}{\mathbf{p}^{2}-\mathbf{k}^{2}+i \varepsilon} \\
& {\left[\frac{\left|\mathbf{k}^{2}-\mathbf{p}^{2}\right|\left(\mathbf{k}^{2}-\mathbf{p}^{2}\right)}{\left(\mathbf{k}^{2}\right)^{2}+\left(\mathbf{p}^{2}\right)^{2}-2 \mathbf{k}^{2} \mathbf{p}^{2} \cos \theta}-1\right]+(\theta \leftrightarrow \pi-\theta) . }
\end{aligned}
$$

The remaining $\mathbf{k}^{2}$ integration is then divided into two pieces, from 0 to $\Lambda_{I}^{2}$ ( $\mathrm{L}$ region) and from $\Lambda_{I}^{2}$ to $\Lambda_{0}^{2} \rightarrow \infty$ (H sector). In the L part, using

$$
\frac{w_{p}^{2}}{w_{k}}=m\left(1+\frac{\mathbf{p}^{2}}{m^{2}}\right)\left[1-\frac{\mathbf{k}^{2}}{2 m^{2}}+\frac{3\left(\mathbf{k}^{2}\right)^{2}}{8 m^{4}}+\ldots\right]
$$

and keeping terms up to order $\eta^{2}$, one obtains

$$
\begin{aligned}
A_{L}^{(b) d i r} \simeq-\frac{2 e^{4}}{\pi \Theta^{2}} m\{ & \left(1+\frac{\mathbf{p}^{2}}{2 m^{2}}\right)[\ln (2|\sin \theta|)+i \pi]-\frac{\mathbf{p}^{2}}{m^{2}} \\
& \left.-\frac{1}{2} \cos \theta \ln \left(\frac{1-\cos \theta}{1+\cos \theta}\right) \frac{\mathbf{p}^{2}}{m^{2}}-\left(1-2 \cos ^{2} \theta\right) \frac{\mathbf{p}^{4}}{\Lambda_{I}^{4}}\right\} .
\end{aligned}
$$

In the $\mathrm{H}$ region, the integrand is replaced by its Taylor expansion around $\mathbf{p}^{2}=0$ which is given by

$$
\left[-\frac{2 m^{2} \cos \theta}{\left(\mathbf{k}^{2}\right)^{2} \sqrt{\mathbf{k}^{2}+m^{2}}}\right] \mathbf{p}^{2}+\left[\frac{2 m^{2}\left(1-2 \cos ^{2} \theta\right)}{\left(\mathbf{k}^{2}\right)^{3} \sqrt{\mathbf{k}^{2}+m^{2}}}-\frac{2 \cos \theta \sqrt{\mathbf{k}^{2}+m^{2}}}{\left(\mathbf{k}^{2}\right)^{3}}\right] \mathbf{p}^{4}+\mathcal{O}\left(\mathbf{p}^{6}\right) .
$$

Performing the $\mathbf{k}^{2}$ integrations one obtains, up to order $\eta^{2}$,

$$
A_{H}^{(b) d i r} \simeq-\frac{2 e^{4}}{\pi \Theta^{2}} m\left\{\left(1-2 \cos ^{2} \theta\right) \frac{\mathbf{p}^{4}}{\Lambda_{I}^{4}}\right\} .
$$

Adding (3.10) and (3.11), we get

$$
\begin{aligned}
A^{(b) d i r} \simeq-\frac{2 e^{4}}{\pi \Theta^{2}} m\{ & \left(1+\frac{\mathbf{p}^{2}}{2 m^{2}}\right)[\ln (2|\sin \theta|)+i \pi]-\frac{\mathbf{p}^{2}}{m^{2}} \\
& \left.-\frac{1}{2} \cos \theta \ln \left(\frac{1-\cos \theta}{1+\cos \theta}\right) \frac{\mathbf{p}^{2}}{m^{2}}\right\}
\end{aligned}
$$


The $k^{0}$ integration of the twisted box diagram, the second in Fig. 5(b), gives

$$
\begin{aligned}
A^{(b) t w i s t}=-\frac{e^{4}}{2 \pi^{2} \Theta^{2}} \int d^{2} \mathbf{k} & \left(\frac{w_{k} w_{k-s}-w_{p}^{2}}{w_{k} w_{k-s}\left(w_{k}+w_{k-s}\right)}\right) \\
& {\left[\frac{(\mathbf{k} \times \mathbf{s})^{2}-\left(\mathbf{p} \times \mathbf{p}^{\prime}\right)^{2}}{(\mathbf{k}-\mathbf{p})^{2}\left(\mathbf{k}-\mathbf{p}^{\prime}\right)^{2}}\right]+\left[\mathbf{p}^{\prime} \leftrightarrow-\mathbf{p}^{\prime}\right] }
\end{aligned}
$$

where $\mathbf{s}=\mathbf{p}+\mathbf{p}^{\prime}$ and we recall that $w_{k-s}=\sqrt{(\mathbf{k}-\mathbf{s})^{2}+m^{2}}$. This integration has a rather non trivial angular part but the use of the approximation procedure before performing it allows analytical calculations and one ends up, after adding its final particles exchanged partner, with

$$
A_{L}^{(b) t w i s t} \simeq 0 \text { and } A^{(b) t w i s t} \simeq A_{H}^{(b) t w i s t} \simeq-\frac{2 e^{4}}{\pi \Theta^{2}} m\left\{\frac{\mathbf{p}^{2}}{2 m^{2}}\right\} .
$$

Thus, the total box amplitude, $A^{(b)}=A^{(b) d i r}+A^{(b) t w i s t}$, is finite and, up to order $\mathbf{p}^{2} / m^{2}$, is given by

$$
\begin{aligned}
A^{(b)} \simeq-\frac{2 e^{4}}{\pi \Theta^{2}} m\{ & \left(1+\frac{\mathbf{p}^{2}}{2 m^{2}}\right)[\ln (2|\sin \theta|)+i \pi]-\frac{\mathbf{p}^{2}}{2 m^{2}} \\
& \left.-\frac{1}{2} \cos \theta \ln \left(\frac{1-\cos \theta}{1+\cos \theta}\right) \frac{\mathbf{p}^{2}}{m^{2}}\right\} .
\end{aligned}
$$

Notice that, as a by product, this cutoff procedure gives the origin (whether from the L or $\mathrm{H}$ sectors) of each contribution. One sees, for example, that the contribution of the twisted box diagram comes entirely from $\mathrm{H}$ energy which is naturally expected from a graph that involves backward propagation in time.

The third group, the seagull scattering, has to be treated more carefully since it carries the divergence of the four point function. One can immediately see that each of the $k^{0}$ integrations of the the gauge bubble and the two triangle diagrams would diverge if made separately. However, taking all the diagrams of group $(c)$ together, the divergences of the $k^{0}$ integrations cancel out identically and the angular integrations, which again are linear combinations of $\mathrm{I}_{n}$, lead to

$$
\begin{aligned}
A^{(c)}=-\frac{e^{4}}{2 \pi \Theta^{2}}\left\{\int d\left(\mathbf{k}^{2}\right)\left(\frac{w_{p}^{2}+w_{k}^{2}}{w_{k}}\right) \frac{\operatorname{sgn}\left(\mathbf{k}^{2}-\mathbf{p}^{2}\right)\left(\mathbf{k}^{2}-\cos \theta \mathbf{p}^{2}\right)}{\left(\mathbf{k}^{2}\right)^{2}+\left(\mathbf{p}^{2}\right)^{2}-2 \mathbf{k}^{2} \mathbf{p}^{2} \cos \theta}\right. \\
\left.-\int d\left(\mathbf{k}^{2}\right) \frac{1}{w_{k}}\left[\frac{\left|\mathbf{k}^{2}-\mathbf{p}^{2}\right|\left(\mathbf{k}^{2}-\mathbf{p}^{2}\right)}{\left(\mathbf{k}^{2}\right)^{2}+\left(\mathbf{p}^{2}\right)^{2}-2 \mathbf{k}^{2} \mathbf{p}^{2} \cos \theta}-1\right]\right\}+(\theta \leftrightarrow \pi-\theta) .
\end{aligned}
$$


Repeating the procedure exemplified with the box diagram one finds

$$
\begin{aligned}
A_{L}^{(c)} \simeq-\frac{2 e^{4}}{\pi \Theta^{2}} m\{ & \left(1+\frac{\mathbf{p}^{2}}{2 m^{2}}\right)\left[\ln \left(\frac{\Lambda_{I}^{2}}{\mathbf{p}^{2}}\right)-\ln (2|\sin \theta|)\right]+\frac{\mathbf{p}^{2}}{m^{2}} \\
& \left.+\frac{1}{2} \cos \theta \ln \left(\frac{1-\cos \theta}{1+\cos \theta}\right) \frac{\mathbf{p}^{2}}{m^{2}}+\frac{1}{2}\left(1-2 \cos ^{2} \theta\right) \frac{\mathbf{p}^{4}}{\Lambda_{I}^{4}}+\frac{\Lambda_{I}^{4}}{16 m^{4}}\right\}, \\
A_{H}^{(c)} \simeq-\frac{2 e^{4}}{\pi \Theta^{2}} m\{- & \left(1+\frac{\mathbf{p}^{2}}{2 m^{2}}\right) \ln \left(\frac{\Lambda_{I}^{2}}{4 m^{2}}\right)-1 \\
& \left.-\frac{1}{2}\left(1-2 \cos ^{2} \theta\right) \frac{\mathbf{p}^{4}}{\Lambda_{I}^{4}}-\frac{\Lambda_{I}^{4}}{16 m^{4}}+\left[\frac{\Lambda_{0}}{m}\right]\right\}
\end{aligned}
$$

and, thus, the total seagull contribution to the amplitude is

$$
\begin{aligned}
A^{(c)} \simeq-\frac{2 e^{4}}{\pi \Theta^{2}} m\{( & \left(+\frac{\mathbf{p}^{2}}{2 m^{2}}\right)\left[\ln \left(\frac{4 m^{2}}{\mathbf{p}^{2}}\right)-\ln (2|\sin \theta|)\right] \\
& \left.-1+\frac{1}{2} \cos \theta \ln \left(\frac{1-\cos \theta}{1+\cos \theta}\right) \frac{\mathbf{p}^{2}}{m^{2}}+\frac{\mathbf{p}^{2}}{m^{2}}\right\}+\frac{2 e^{4}}{\pi \Theta^{2}} \Lambda_{0} .
\end{aligned}
$$

The constant divergent term above can be suppressed by a counterterm of the form $-\frac{2 e^{4}}{\pi \Theta^{2}} \Lambda_{0}\left(\phi^{*} \phi\right)^{2}$ introduced in the Lagrangian density. We can also imagine that the bare self-coupling $\lambda$ carries a divergent part that just cancel the divergence of the four point function. In any case, we take the finite part of (3.19) as the 1-loop renormalized (c) contribution. This would be the result if we had used dimensional renormalization.

The pure CS exchange scattering, the sum $A^{(b)}+A^{(c)}$, is given by

$$
A^{(C S)} \simeq-\frac{2 e^{4}}{\pi \Theta^{2}} m\left\{\left(1+\frac{\mathbf{p}^{2}}{2 m^{2}}\right)\left[\ln \left(\frac{4 m^{2}}{\mathbf{p}^{2}}\right)+i \pi\right]-1+\frac{\mathbf{p}^{2}}{2 m^{2}}\right\},
$$

and it is noticeable that the cancellation of the $\theta$ dependent terms of the box and the seagull amplitudes happens in both dominant and subleading orders.

The total renormalized 1-loop particle-particle scattering amplitude, $A^{(a)}+A^{(C S)}+A^{(p)}+$ $A^{(v)}$, is independent of the scattering angle $\theta$ and, up to order $\mathbf{p}^{2} / m^{2}$, is given by

$$
\begin{aligned}
A^{(1)} \simeq & m\left(\frac{\lambda^{2}}{32 \pi m^{2}}-\frac{2 e^{4}}{\pi \Theta^{2}}\right)\left[\ln \left(\frac{4 m^{2}}{\mathbf{p}^{2}}\right)+i \pi\right] \\
& -m\left(\frac{\lambda^{2}}{32 \pi m^{2}}+\frac{2 e^{4}}{\pi \Theta^{2}}\right) \frac{\mathbf{p}^{2}}{2 m^{2}}\left[\ln \left(\frac{4 m^{2}}{\mathbf{p}^{2}}\right)+i \pi\right] \\
& +m\left(\frac{\lambda^{2}}{8 \pi m^{2}}-\frac{7 e^{4}}{3 \pi \Theta^{2}}\right)-m\left(\frac{\lambda^{2}}{192 \pi m^{2}}+\frac{24 e^{4}}{5 \pi \Theta^{2}}\right) \frac{\mathbf{p}^{2}}{m^{2}} .
\end{aligned}
$$


The leading term of the above expansion, which coincides with the result of Ref. [11], vanishes if the self-interaction parameter is fixed at one of the critical values $\lambda_{c}^{ \pm}= \pm 8 m e^{2} /|\Theta|$ but the subdominant terms do not. The implications of this fact will be discussed next.

\section{RELATIVISTIC CORRECTIONS TO AB SCATTERING}

Prior to any comparison with the nonrelativistic case, the normalization of states has to be properly adjusted. In the relativistic case one takes $\left\langle\mathbf{p}^{\prime} \mid \mathbf{p}\right\rangle=2 w_{p} \delta\left(\mathbf{p}^{\prime}-\mathbf{p}\right)$ while the usual normalization in a NR theory does not have the $2 w_{p}$ factor and thus the CM amplitudes, calculated in the last section, must be multiplied by

$$
\left(\frac{1}{\sqrt{2 w_{p}}}\right)^{4}=\frac{1}{4 m^{2}}\left[1-\frac{\mathbf{p}^{2}}{m^{2}}+\ldots\right] .
$$

The tree level and the 1-loop amplitudes, equations (3.1) and (3.21), are then rewritten, up to order $\mathbf{p}^{2} / m^{2}$, as

$$
\mathcal{A}^{(0)}=-\frac{\lambda}{4 m^{2}}-i \frac{2 e^{2}}{m \Theta} \cot \theta+\left[\frac{\lambda}{4 m^{2}}+i \frac{e^{2}}{m \Theta} \cot \theta\right] \frac{\mathbf{p}^{2}}{m^{2}}
$$

and

$$
\begin{aligned}
\mathcal{A}^{(1)} \simeq \frac{m}{8 \pi} & \left(\frac{\lambda^{2}}{16 m^{4}}-\frac{4 e^{4}}{m^{2} \Theta^{2}}\right)\left[\ln \left(\frac{4 m^{2}}{\mathbf{p}^{2}}\right)+i \pi\right] \\
& -\frac{m}{8 \pi}\left(\frac{3 \lambda^{2}}{32 m^{4}}-\frac{2 e^{4}}{m^{2} \Theta^{2}}\right) \frac{\mathbf{p}^{2}}{2 m^{2}}\left[\ln \left(\frac{4 m^{2}}{\mathbf{p}^{2}}\right)+i \pi\right] \\
& +\frac{m}{8 \pi}\left(\frac{\lambda^{2}}{4 m^{4}}-\frac{14 e^{4}}{3 m^{2} \Theta^{2}}\right)-\frac{m}{8 \pi}\left(\frac{25 \lambda^{2}}{96 m^{4}}+\frac{74 e^{4}}{15 m^{2} \Theta^{2}}\right) \frac{\mathbf{p}^{2}}{m^{2}},
\end{aligned}
$$

where calligraphic $\mathcal{A}$ means that the amplitude is written in the nonrelativistic normalization.

Confronting the tree levels, of the relativistic and the NR scattering amplitudes, one sees that the self-interaction parameters are related by $v=\lambda / 4 m^{2}$ and the critical values for which the 1-loop NR and leading relativistic scattering amplitudes vanish are also related by 


$$
v_{c}^{ \pm}=\frac{\lambda_{c}^{ \pm}}{4 m^{2}}= \pm \frac{2 e^{2}}{m|\Theta|}= \pm \frac{4 \pi}{m} \alpha \operatorname{sgn}(\alpha),
$$

where the $\mathrm{AB}$ parameter is $\alpha=e^{2} / 2 \pi \Theta$. By choosing the value $v_{c}^{+}$, corresponding to a repulsive contact interaction, the tree amplitude reduces, after multiplying by the appropriated kinematical factor, to the Aharonov-Bohm amplitude for identical particles (1.4). The leading order vanishes at $v_{c}^{+}$whereas the subdominant terms that survive, namely

$$
\begin{aligned}
\mathcal{A}^{\mathrm{sub}}= & \frac{4 \pi}{m} \alpha\left[\operatorname{sgn}(\alpha)+\frac{i}{2} \cot \theta\right] \frac{\mathbf{p}^{2}}{m^{2}}+\frac{17}{3} \frac{\pi}{m} \alpha^{2} \\
& -\frac{\pi}{m} \alpha^{2} \frac{\mathbf{p}^{2}}{m^{2}}\left[\ln \left(\frac{4 m^{2}}{\mathbf{p}^{2}}\right)+i \pi\right]-\frac{54}{5} \frac{\pi}{m} \alpha^{2} \frac{\mathbf{p}^{2}}{m^{2}}
\end{aligned}
$$

represent relativistic corrections to the Aharonov-Bohm scattering.

Part of the correction of the tree level $(\sim \alpha)$ is due to the normalization of states and so has a pure kinematical origin, but not all of it since the scattering amplitude corresponding to the exchange of one virtual gauge particle depends on the CM energy as a consequence of the minimal coupling. The other corrections come from the 1-loop $\left(e^{4}\right)$ contribution to the perturbative expansion and are indeed relativistic. These kind of terms, proportional to $\alpha^{2}$, do not exists in nonrelativistic AB scattering (which exact result is function of $\sin \alpha$ ) and then may be detected in experiments with fast particles. The relativistic correction has non trivial contributions (the third parcel of (4.5), for example) that can only be incorporated in a nonrelativistic framework through the addition of new nonrenormalizable interactions to the Lagrangian (1.1). This aspect will be discussed elsewhere. 


\section{Acknowledgements}

This work was partially supported by Conselho Nacional de Desenvolvimento Científico e

Tecnológico (CNPq) e Fundação de Amparo à Pesquisa do Estado de São Paulo (FAPESP). 


\section{REFERENCES}

[1] Y. Aharonov and D. Bohm, Phys. Rev. 115 (1959) 485; for a review see S. Ruijsenaars, Ann. Phys. (N.Y.) 146 (1983) 1.

[2] J. M. Leinaas and J. Myrheim, Nuovo Cimento 37B (1977) 1.

[3] F. Wilczek, Phys. Rev. Lett. 48 (1982) 1144; Phys. Rev. Lett. 49 (1982) 957.

[4] D. P. Arovas, R. Schrieffer, F. Wilczek and A. Zee, Nucl. Phys. B251 (1985) 117.

[5] G. Amelino-Camelia, Phys. Lett. B326 (1994) 282; Phys. Rev. D51 (1995) 2000; C. Manuel and R Tarrach, Phys. Lett. B328 (1994) 113 ; S. Ouvry, Phys. Rev. D50 (1994) 5296; C. R. Hagen, Phys. Rev. D52 (1995) 2466; P. Giacconi, F. Maltoni and R. Soldati, Phys. Rev. D53 (1996) 952.

[6] C. R. Hagen, Phys. Rev. D31 (1985) 848.

[7] R. Jackiw and S. Y. Pi, Phys. Rev. D42 (1990) 3500.

[8] O. Bergman and G. Losano, Ann. Phys. (N.Y.) 229 (1994) 416.

[9] D. Z. Freedman, G. Losano and N. Rius, Phys. Rev. D49 (1994) 1054.

[10] S. -J. Kim, Phys. Lett. B343 (1995) 244.

[11] M. Boz, V. Fainberg and N. K. Pak, Phys. Lett. A207 (1995) 1; Ann. Phys. (N.Y.) 246 (1996) 347 .

[12] M. Gomes, J. M. C. Malbouisson and A. J. da Silva, "On the Nonrelativistic Limit of the $\lambda \phi^{4}$ Theory in 2+1 Dimensions", IFUSP/P-1244 (1996), hep-th/9610247. 


\section{Figure captions}

Fig. 1 - Vacuum polarization correction in one loop order.

Fig. 2 - One loop correction to the trilinear vertex.

Fig. 3 - Tree level scattering.

Fig. 4 - Basic mixing interactions diagrams .

Fig. 5 - One loop order particle-particle scattering. In the momenta assignment shown, $s=p_{1}+p_{2}, q=p_{1}-p_{1}^{\prime}$ and $u=p_{1}^{\prime}-p_{2}$. 